\title{
Electric, thermal, and magnetic based digital interfaces for next generation food experiences
}

Kasun Karunanayaka $^{1 *}$, Adrian David Cheok ${ }^{1,2}$, Nur Amira Samshir ${ }^{1}$, Nurafiqah Johari ${ }^{1}$, Surina Hariri ${ }^{1}$, Nur Ellyza Abd Rahman ${ }^{1}$, Nur Ain Mustafa and Prem Anton ${ }^{1}$

${ }^{1}$ Imagineering Institute, Nusajaya, Malaysia

${ }^{2}$ City University London, UK

\begin{abstract}
In this article we present how electrical, magnetic, and thermal interfaces can create and modify taste and smell sensations with new interactions. In future, these technologies can play a vital role in food consumption, food manufacturing, food based interactions and multisensory communication. We provide a detail review of previous electrical, thermal and magnetic interfaces in the related research area and then we present the currently ongoing research works carried out by the authors. We also discuss how these technologies can create, modify new interactions for industry of foods, marketing, communication and computing. Especially we believe that the future of internet will not only depend with visual, audio, and tactile stimuli but also with smell (olfaction) and taste (gustation). Humans would share these stimuli collectively as an experience digitally, like they do with the visual and audio media on the internet. We want to propose an idea of digital olfaction and gustation as input and output for interaction for creating and experiencing digital representation of foods.
\end{abstract}

\section{Introduction}

Computers and the Internet have both changed people's life style so rapidly in last few decades. Today we are moving towards a hyper connected era where humans and all the electronic devices that we use will be connected together and form a single network. Most of the machines will be also equipped with human senses such as audio visual and tactile. Therefore, we believe the next important breakthrough of internet would be the use of smell and taste digitally. This would include sensing smell and taste information from one location, transferring them over the internet digitally and effectively regenerate the signals at the destination. Since internet works on digital data, we need the sensors to sense smell and taste information and convert them to digital for communication. The destination receiving those data needs to convert from digital signals to analog smell and taste sensations. Therefore, there is a need for smell and taste actuation technologies to be compatible with digital information. Since smell and taste detection sensors and products are already available, actuation becomes the most important. To solve this issue, over the years we have developed and proposed number of actuation technologies that could regenerate the smell and taste experiences. These interfaces are developed using three main technologies.

1) Electrical Interfaces - Electric Sour Taste Interface, Electric Smell Interface

2) Thermal Interfaces - Thermal Sweet Taste Interface

3) Magnetic Interfaces - Magnetic Food and Magnetic Levitation Platform

Electric sour taste interface is an electrical tongue actuator stimulation device, which the user places in their mouth and controlled by the computer where it can effectively generate sour sensations. Our previous user studies have suggested by changing the current and frequency we can also generate salt and bitter sensations. Electric smell interface is an attempt to stimulate smell sensation by stimulating the human olfactory receptors using weak electrical pulses. There will be two thin electrodes going to be placed on the nasal concha for stimulation.

Thermal sweet taste interface uses a copper electrode that changes the temperature over the time. According to the previous literatures and our pilot user studies, while the device is on a high temperature rise, users feel a sweet taste.

Magnetic food research is focusing on developing edible magnetically reactive foods. Most of the iron enriched foods that we consume in our daily life are magnetically reactive. Therefore, we are looking in to use iron and use some other magnetically reactive metals to mix with foods and make them edible. Then we will be able implement new interactions to these foods like change the weight of the food, turn, rotate and levitate. Magnetic Table Interface is going to be made with an array of Bitter electromagnets [32] underneath the table. Then using the magnetic flux generates from this interface, we will be able to introduce new interactions for the cutleries and foods such as change of the weight, rotate and levitate.

We have conceptualized some scenarios for how these smell and taste interfaces would work in future scenarios. Our ideas are

Correspondence to: Kasun Thejitha Karunanayaka, Research Fellow, Imagineering Institute (IDM Lab Sdn Bhd), Anchor 5, Mall of Medini, No. 4 Lebuh Medini Utara, Nusajaya, 79250, Johor, Malaysia, Tel: +607 509 6716; +60 17358 2163; Fax: +607 509 6713; E-mail: kasun@imagineeringinstitute.org

Key words: digital taste, digital smell, multi-sensory communication, remote foods, food levitation, food technologies

Received: December 20, 2015; Accepted: January 04, 2016; Published: January 07, 2016 
virtual SMS menu, culinary education, collaborative remote dining, ambiguous food, and approximating a service for future food printing at home.

Food menu App: Internet and mobile phone services can be integrated with our smell-taste technologies to provide smell and taste information for their digital contents. For example, when a restaurant introduces a new menu, they can conduct a special promotion through their mobile App using the smell-taste accessories. They can send online coupons to their customers containing smell-taste information for new dish and when the customer begins to read this message, he or she can smell or taste the new menu from their accessories. Customers are able to check the restaurant menu using the smell-taste accessory by logging into the App, before visiting the restaurant physically.

Culinary education: Watching a recipe video gives us two sources of information visual and auditory. In the near future we could see audio visual systems that are expanded to utilize smell and taste. You can check your own dish in progress by comparing the taste to the chef's dish. Similarly, we could add new dimensions to food entertainment media, when the lid comes off a steaming pot on your streaming video; you experience the same smell and taste sensation.

New food dining experiences: Using the magnetic table platform and magnetic foods users will be able to experience new food interactions in future. After users order foods in the restaurants they will be able to adjust the weight of the utensils such as spoon, fork, knife and plates. For example they will be able to change the weight of these utensils to zero (to simulate zero gravity environment) or minus (negative gravity) where they can levitate them with the foods. Foods served in the restaurant can be also magnetically active. This enables adding different properties to the foods like change the shape and texture of the food, changing the weight of the food and utensils, vibration, repulsion, attraction, and levitation.

Collaborative remote dining: We would be able to enhance remote co-dining and co-cooking experience, by translating tasting sense to music notes to record our daily recipe in an interesting way. We call this "Taste symphony". For example capturing the taste from a curry cooked at one location and reconstructing and delivering in a personalize manner, in one scenario we envision a simple consumer technology that allows for these lonely individuals to cook meals collaboratively with their children remotely. By enhancing the taste generated in the kitchen, using a protocol to transmit taste and associate the ability of reconstructing the taste with the remote collaborative cooking space would recombine the creative nature of the humans all over the world. It will give positive thinking of remote co-presence and co-living experience through this new kitchen space.

Internet food: Sensing occurs between the sender's environment and the media. The sensors can detect smells and tastes from the environment or from specific foods. An example is that the various sensors in a kitchen can measure the smells and tastes currently in the room. The sensed aromas and tastes can be communicated through internet after converting them into digital information and actuate on a specific user using the small wearable device. The $3 \mathrm{D}$ food printing could be the ultimate output, eventually. A chef could use the taste and smell to give quick feedback of an ongoing recipe which when finalized could be sent to a 3D printing food. In the future we'd expect a home user to have such devices installed in their homes like with paper printer. A user could design his / her own food to be printed and send it to his / her friends printing device. While constructing the food to save resource they can approximate the food with taste and smell device. They could even upload this as a work in progress to allow other to quickly sample the food for approval.

The next sections of this paper will discuss the literature related to these interfaces, development details of our new interfaces and their applicability for various different fields.

\section{Literature review of previous digital interfaces for foods}

New user interfaces that we are developing for the smell and taste uses electrical energy, magnetic energy and thermal energy. The following subsections detail the previous works related to our research prototypes.

\section{Electrical interfaces}

In one of his experiments, Alessandro Volta put two coins, made of different metals, on both sides of his tongue (up and down) and connected them through a wire. He mentioned that he felt a salty sensation [1]. In 1976, Plattig and Innitzer were electrically stimulated a single human tongue papillae with a silver electrode using five young subjects [2]. They used both negative and positive electrical pulses with a frequency range of 50 to 800 Hertz. The results provided effective responses for the sour taste $(22.2 \%)$ and some small responses for the bitter (3.8\%) and salty (1.8\%) sensations. When we consider about the taste, mathematical models of the excitation for chemical stimulation of the taste receptors have been proposed in 1977 [3]. Lawless presented metallic taste generation from electrical and chemical stimulation [4]. They observed the similarities and differences of stimulation with metals, electrical stimulation, and solutions of divalent salts and ferrous sulphate and investigated sensations that occurred across oral locations using electrical stimulation and different metal anodes and cathodes.

The electrical stimulated sour taste experience was recorded in 1996 from weak electrical current delivered to the surface of the tongue [5]. However, recent results have shown that electrogustronomy relates to an ability to appreciate all the four tastes: sour, sweet, salt, and bitter. The electrogustrometry correlated with all four taste qualities was found in 2007 [6]. They proposed that there is a direct depolarizing mode of action of the sensory-neural tongue aspects.

Nakamura and Miyashita has used electricity for augmented gustation [7]. They apply electric current through isotonic drinks and juicy foods to change the taste perception by pulsing voltage and amplitude input. It has recently, produced what they call olfactory hallucinations [8] by placing nodes on the ventral surface of the frontal lobe of children with epilepsy. 11 out of 16 patients stated that they were able to smell perceptions.

In most of the olfactory system related studies examining electrical activity of the olfactory bulb, an adequate olfactory stimulus such as blowing odorous air into the nose has been used as a routine method of activating the olfactory bulb. Only few attempts have been made to do an electrical stimulation of the olfactory system. Yamamoto has stimulated the human olfactory mucosa by electrical pulse to detect the bulbar potentials [9]. Electrical stimulation $(2 \mathrm{~mA}, 0.5 \mathrm{~ms})$ of the human olfactory mucosa evoked a change in potential recorded from the frontal sector of the head. During an experiment conducted in 1997, the properties of the olfactory bulb potential evoked by electrical stimulation of the olfactory mucosa were studied in rabbits immobilized with d-tubocurarine [10]. The evoked potential was a slow negative wave when recorded from the surface of the bulb. In 2002 they concluded that electrical olfactory evoked potential (EOEP) is suitable for electrophysiology [11]. The relationship between the EOEP and 
Toyoda and Takagi's perfumist's strip method [12] which is a standard Japanese means of psychophysical olfactometry are investigated. Electrical stimulation via bipolar electrodes $(2 \mathrm{~mA}, 0.5 \mathrm{~ms}, 300$ trials $)$ is feed to the olfactory mucosa. Four channels of EOEP are amplified, filtered (2 to $250 \mathrm{~Hz}$ ) and recorded. During electrical stimulation of right or left of the olfactory mucosa evoked an electrical olfactory evoked potential. However, there is no sense of smell occurred.

From the above, the possibility of using non-chemical stimulation methods to stimulate smell and taste sensations digitally can be seen. However, the above reported studies are conducted mainly in the medical domain (with controlled environments), are invasive, or only in the experimental stage. Therefore, to achieve electrical and magnetic stimulation methods as a means of actuating the sensations of taste and smell, this research will achieve research breakthroughs in controllability, accuracy, and robustness.

\section{Thermal interfaces}

When we consider about multisensory communication, sensing and actuation of sweet taste digitally is an extremely important requirement. Almost all the sweet taste interfaces developed earlier in the field of Human Computer Interaction, were using chemicals to produce sweet sensations [13-15] (Kortum, 2008 ; MaynesAminzade,2005; Edition.cnn.com, 2015). By referring to some prior works we have identified that thermal stimulation of TRPM 5 channel (Transient receptor potential cation channel subfamily $\mathrm{M}$ member 5) [16] resulted in sweet sensations [17]. The previous experiment showed that rapid heating and cooling between $15^{\circ} \mathrm{C}$ to $35^{\circ} \mathrm{C}$ (Temperature was varied at approximately $\left.\pm 1.5^{\circ} \mathrm{C} \mathrm{s}^{-1}\right)$ can evoke sweetness.

According to the paper "Heat activation of TRPM5 underlies thermal sensitivity of sweet taste" [16] defines that increasing the temperature resulted activation of TRPM 5 channel that generates a depolarizing potential in the taste receptor cells. This effect causes the enhanced sweetness perception at high temperatures and "thermal taste", the phenomenon whereby heating or cooling of the tongue evokes sensations of taste in the absence of chemical tastants.

"Affecting Tumbler" [18] is a system that can influence flavor observation. Without modifying the actual flavor of the drink in this research researchers change the temperature of the skin near the nasal area while drinking. Their studies suggested that the flavor richness and aftertaste strength can be significantly improved by heating up the skin in the nasal region.

Even though thermal effect with TRPM5 channel is well studied in the medical field, a proper user interface for HCI which uses this effect to generate/enhance sweet sensations has yet to be developed.

\section{Magnetic interfaces}

Due to the nature of the magnetic fields, direct human interaction is not easily possible because humans cannot readily touch or feel them physically. Even though, magnetic fields are intangible, they could be transformed into different forms of energies or signals to provide tangible interactions. It is possible to generate attraction, repulsion, actuation, kinaesthetic feelings [19] pressure [20,21] using magnetic fields. Further, magnetic materials can be used to develop interactive user interfaces [22-24].

One of the earlier works of the authors, Liquid Interface [25] related to the magnetic table and foods research (Four different prototypes of Liquid Interfaces are shown in Figure 1. This was an Organic User Interface (OUI) [26] that combines Hall Effect sensing and actuation

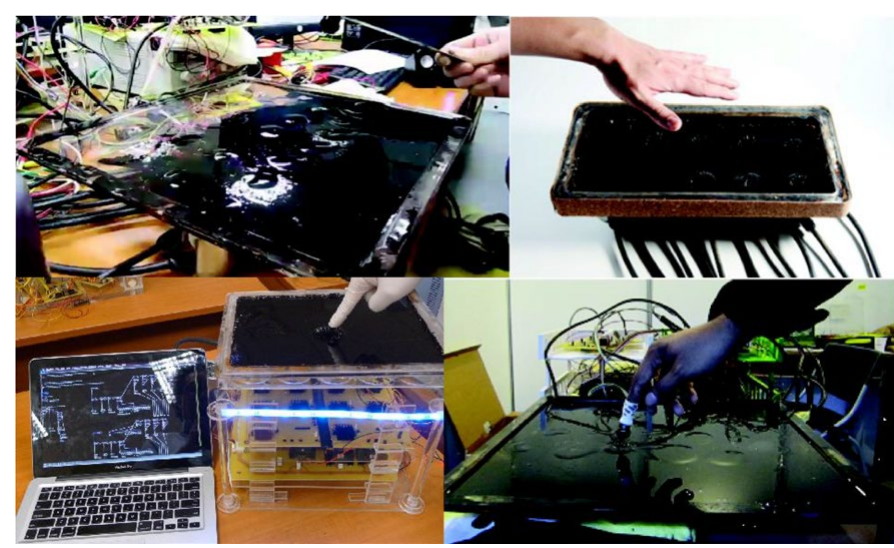

Figure 1. Four different prototypes of the Liquid Interfaces system (a. playing as a piano using a magnetic wand, $b$. touch less interface waving the hand on top of the surface to create and modify ferrofluid patterns, c. direct touch interface that can create and feel ferroflid bubbles, d. playing with piano using a magnetic finger extension).

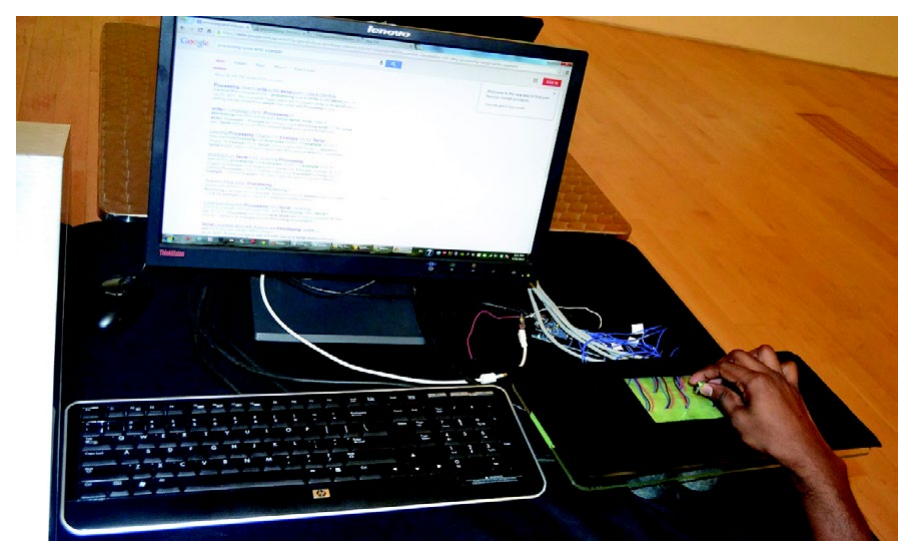

Figure 2. The magnetic table research.

through electromagnetically-manipulated ferrofluid. The movement of magnets worn on the fingertips, over a surface embedded with a Hall Effect sensor array and electromagnets, gives user the ability to interact with the ferrofluid. This system provided a three-dimensional, physically animated response, as well as three-dimensional, spatialsensing inputs. The vibration of the magnets worn on the fingertips, produced by the repulsing polarity of the electromagnets, provides the user with haptic feedback. Liquid Interface is a multimodal interface with a visual, audio and haptic experience. Input and output interactions of this interface are both coupled into a single display. Further, shape changeable surface was developed using a malleable material (ferrofluid) where the user could control and repeat the user interactions. These types of interface will be useful to operate in dynamic environments where the user interface should adapt itself to the external signals.

Haptic mouse [27] (Figure 2) was another similar work done by authors similar to the magnetic table research. This was a pointing interface for computers, which provides mouse functionalities with near surface haptic sensations. These functionalities were attained by tracking the $3 \mathrm{D}$ position of a neodymium magnet using Hall

Effect sensors grid and generating like polarity haptic feedback using an array of electromagnets. Haptic Mouse couples the nearsurface sensing and the haptic-actuation together. It brings haptic 
sensations to $3 \mathrm{D}$ spaces near to the surface which can be considered as a novel experience. Users are able to manipulate the objects in the screen by moving their finger on or above the haptic mouse surface. This added an extra dimension as the input. Haptic sensations can be felt on top of the surface as well as above the surface. Different vibration Pattern based feedback allows this interface to be used as a haptic display.

\section{Our current and work in progress prototypes}

This section will detail the smell, taste and food research works that are being developed by the authors related to digital actuation of smell and tastes. These interfaces are electric sour taste interface, electric smell interface, and thermal sweet taste interface, reproducing smell sensations using magnetic stimulation, magnetic food and magnetic levitation platform.

\section{Electric interfaces}

To generate taste sensations digitally, we developed a prototype that can deliver electric current in a controllable and safe manner to a user's tongue. Our approach can be seen in Figure 3, where a user places the apparatus across the surface of their tongue. We generate an output from a digital source; apply the electric current, which is then outputted to the tongue membrane when the tongue makes physically contact with the device. The current travels across the tongue which excites the taste cells that signal the brain that a taste is being sensed.

To implement the device we created a digital circuit PCB as shown in Figure 4. The hardware consists of a microcontroller which accepts input from a PC terminal via USB connection. The microcontroller outputs Pulse Width Modulation signals (PWM). We use this feature to produce square waves of varying frequencies and magnitudes to control the taste interface. The user feels this current simulation with

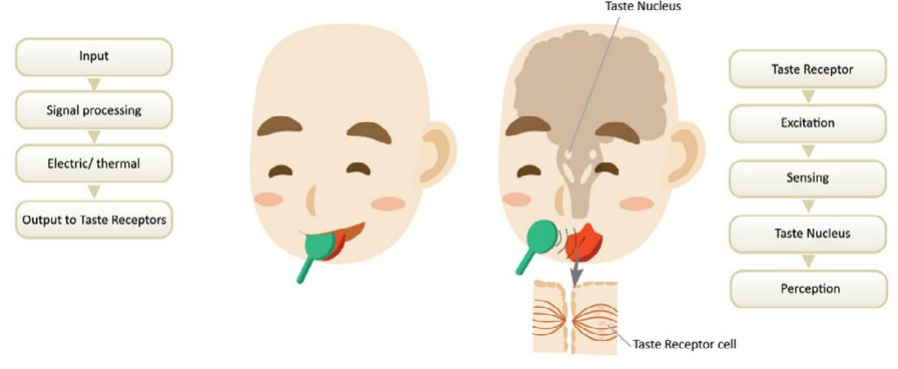

Figure 3. Approach to Digital Taste Stimulation.

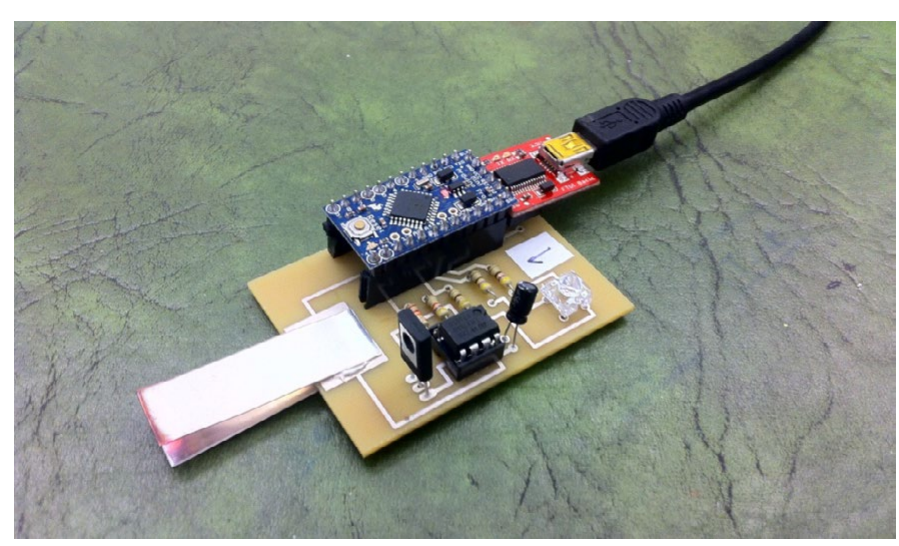

Figure 4. Digital taste interface.

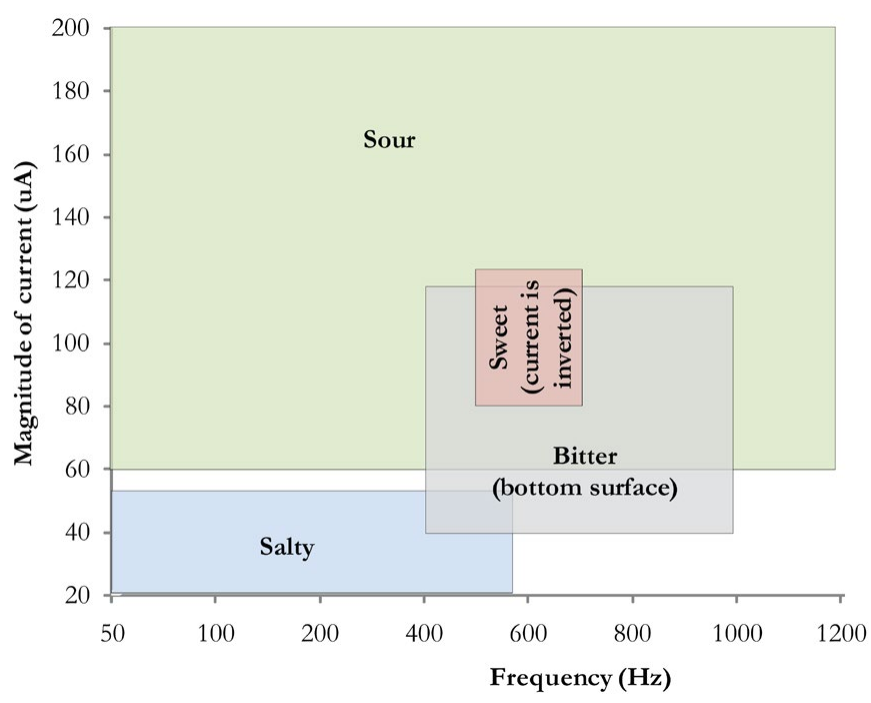

Figure 5. Magnitude and fequency results on taste perception.

their tongue and the result produces several kinds of taste sensations. We use a magnitude range of $20 \mu \mathrm{F}$ to $200 \mu \mathrm{F}$ and a frequency range of $50 \mathrm{~Hz}$ to $1 \mathrm{KHz}$.

Because of impedance differences of everyone's tongues we created an additional part of the circuit that pro-vides a constant current source using an operational amplifier and an NPN transistor. The current output is delivered to the load, the silver tongue electrodes, which are placed on the top and the bottom of the tongue. We mounted the electrodes to the PCB at a slight angle using a special silver epoxy that conducts current from the PCB pad to the electrodes.

We conducted a preliminary study across fifteen participants to evaluate the effectiveness of digital taste as a means to stimulate taste. Participants chosen were of good health and reported no taste problems and were instructed not to eat, drink, or smoke two hours before the tests. We were interested in the robustness of such a system, such as sensory adaption, controllability of the tastes through a population, and also the comfort of using this system. Our study shows (Figure 5) that some tastes can be effectively actuated (salty, sour and bitter) across our population of users. Sweetness was also reported but didn't have as high of a reported rate as the other three tastes. Users showed some hesitance when placing the device into their mouths due to its appearance.

\section{Electric smell interface}

Instead of using any chemical odorous, we hope that by using weak electrical pulse we can excite the smell receptors and brain. We are currently developing small bipolar stimulating electrodes that can reach the smell receptors near the olfactory bulb and concha through the nostrils. There are three regions inside the nostrils called superior nasal concha, middle nasal concha and inferior nasal concha [28] that are nearest to the olfactory receptors where stimulating electrodes could be placed. The placement of electrodes will be done with a help of a medical expert in a way that electrodes would not come off quickly. These electrodes will be controlled by a specially designed circuit that can deliver up to 2 mili-amperes of current (according to the previous experiments carried out in the medical field) to the smell sensitive cells. Currently, we are developing the controller circuit and stimulation electrodes with adjustable parameters for experimenting our idea with users as shown in Figure 6. 


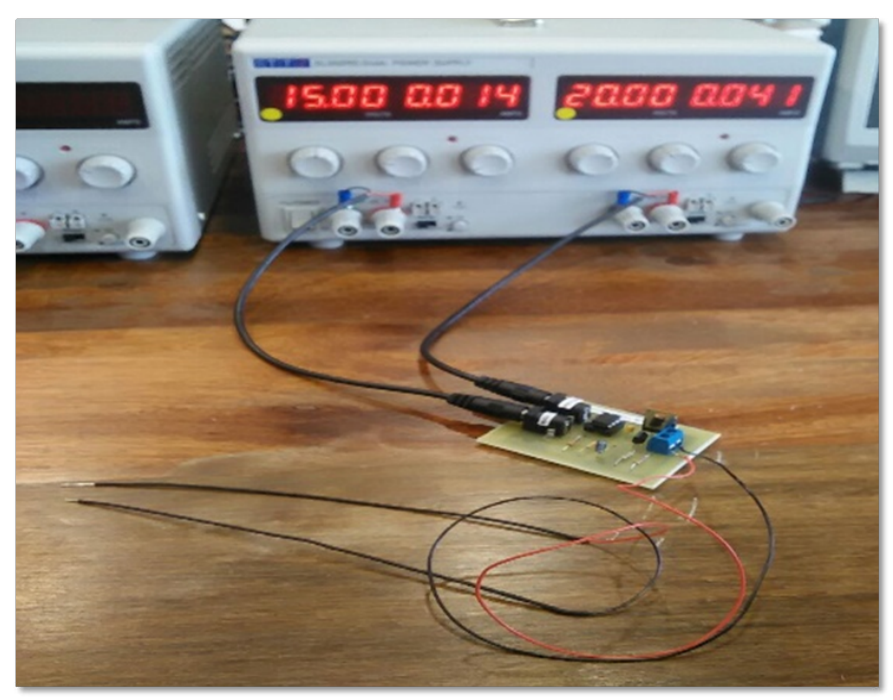

Figure 6. The first prototype of digital smell interface.

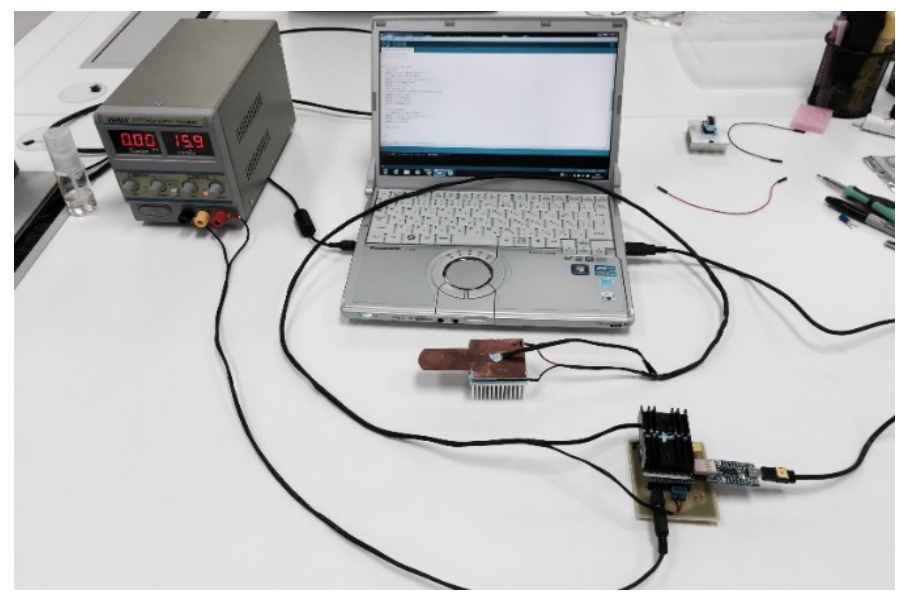

Figure 7. First prototype of the thermal sweet taste interface.

We hope in future, we will be able to develop a robust interface where we can effectively regenerate smell sensations digitally. This digital regeneration of smell will be useful for several industries like gaming, virtual reality, entertainment, online marketing, where people can create content, information, food related to smell that can be shared, learned, and experienced. In the medical industry this research will be useful to treat patients who are suffering from medical conditions like Anosmia and Parosmia.

\section{Thermal interfaces}

\section{Thermal sweet taste interface}

Out of five most fundamental elements of the taste (sweet, sour, bitter, salty and umami) probably sweet taste is the most pleasant out of all the basic tastes [29]. Therefore, we have developed a thermal stimulation device which allows us to produce sweet taste digitally in repeatable and controllable way. When a user using this device he/she will place a copper electrode attached with a Peltier module touching the surface of the tongue and will be able to experience sweet taste sensations. By continuously improving this device we may fulfill people's missing need of sharing taste experiences with others remotely.

Thermal Sweet Taste interface first prototype is shown in Figure 7 and this system consists three main components. Those components are electronic controller circuit; firmware runs in the microcontroller and pc software.

As shown in Figure 8, the controller circuit consists with a microcontroller, copper electrode attached together with a Peltier module, h-bridge motor driver, current sensor, temperature sensor, and FTDI serial interface to communicate with the PC. We apply different temperatures on the tongue by adjusting the temperature of the Peltier module that attached to the copper electrode. Peltier is a special kind of semiconductor device where current flows through it one side becomes heated up while the other side cools down. By alternating direction of the flow of the current we can swap the heating and cooling sides of the Peltier.

The Peltier module we selected can change its surface temperature from $-40^{\circ} \mathrm{C}$ to $80^{\circ} \mathrm{C}$ and consumes up to $8.5 \mathrm{~A}$ of current based on the voltage and duty cycle. Therefore, it is not possible to directly operate a Peltier using microcontroller pins. In order to overcome this issue, we use an $\mathrm{H}$-Bridge motor driver to operate Peltier module. Motor driver receives control signals by reading the PWM pins of the microcontroller and its output pins control the flow of the current to the Peltier module.

Thermal sweet taste device automatically adjusts the temperature of the electrode with the help of temperature sensor and current flow sensor. These two sensors are connected to the microcontroller through input pins. The microcontroller monitors the sensor values and changes the PWM values accordingly to heat up and cool down the Peltier device.

The PC software will allows users to control the device using computer by enabling heating and cooling effects, set thresholds, set stimulation intervals or program stimulation protocols, and etc. This software can be easily modified as an API where we can combine Sweet Taste Interface with other software applications.

Microcontroller firmware is mainly responsible for three tasks; 1 .) Heating and cooling of the copper electrodes according to the signals receives from the PC software 2.) Measure the flow of the current to the Peltier module 3.) Monitoring the temperature of the electrode using temperature sensor and maintain the proper stimulation temperature.

We have conducted two technical experiments to find out the limitations of the Thermal Sweet Taste interface. Figure 9 shows the range of the currents that can be output by the controller circuit

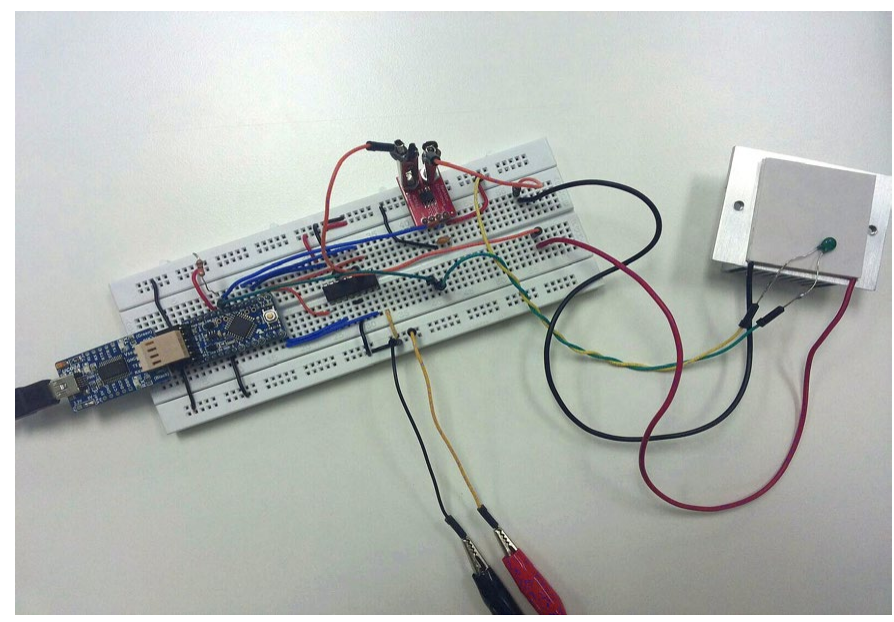

Figure 8. Controller circuit of the thermal sweet taste interface. 


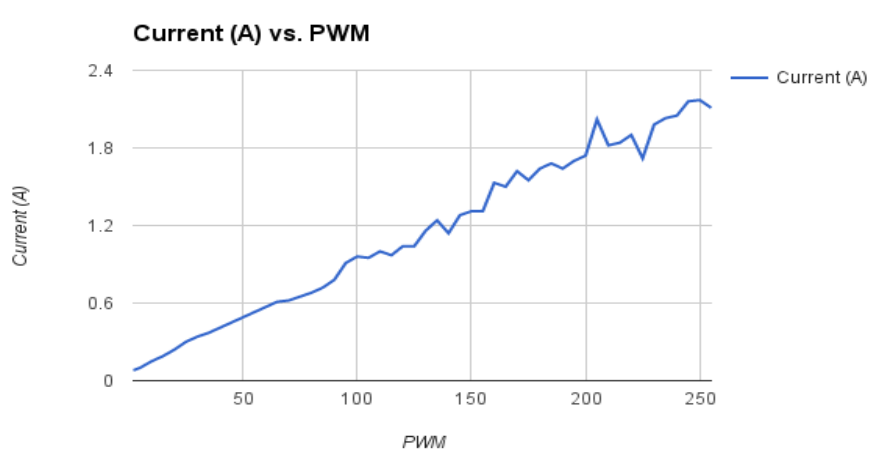

Figure 9. Current output from the Circuit vs. PWM provided.

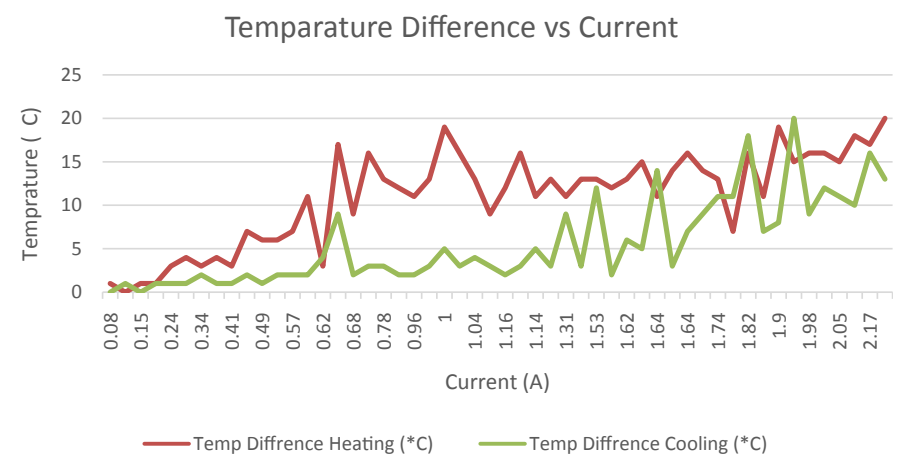

Figure 10. Maximum temperature difference obtained vs. Current input to the Peltier.

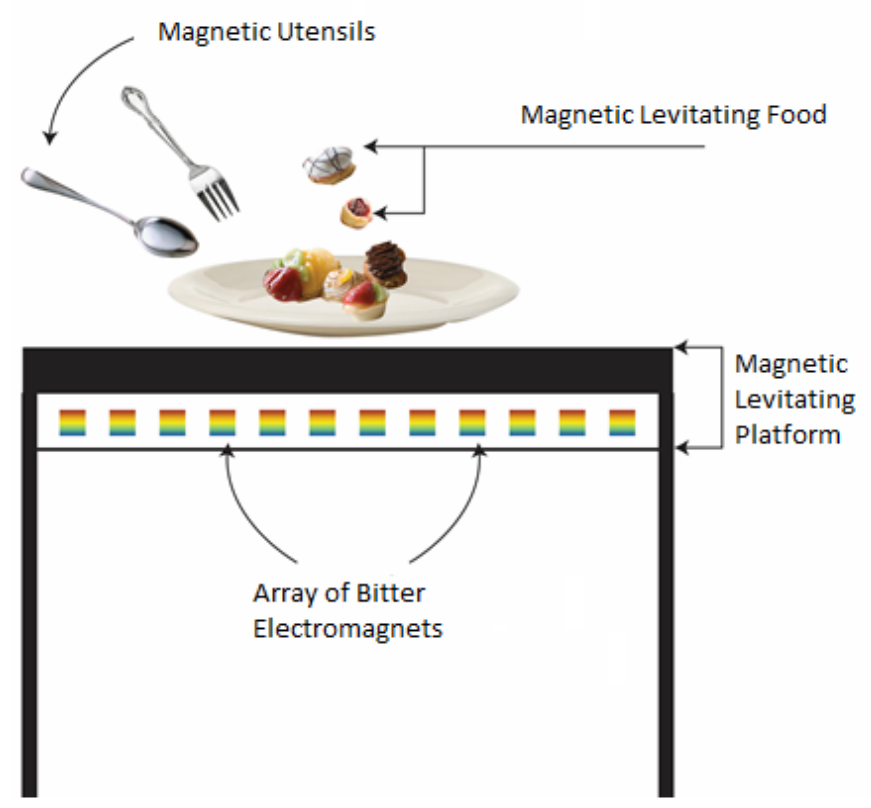

Figure 11. Proposed magnetic table interface.

for different PWM values. The maximum current output gained for the circuit was $2.17 \mathrm{~A}$ for 250 PWM. Figure 10 shows the maximum temperature range that the device can operate while heating and cooling with variable current inputs. We set $25^{\circ} \mathrm{C}$ as the starting temperature and the maximum temperature that we obtained was $45^{\circ} \mathrm{C}$ (for 2.11
A) and minimum temperature was $5^{\circ} \mathrm{C}$ (for $1.72 \mathrm{~A}$ ). We are recently developing the second prototype which achieves faster temperature range. This interface can rise temperature from $25^{\circ} \mathrm{C}$ to $40^{\circ} \mathrm{C}$ within 6.7 seconds and the device consumes $8.5 \mathrm{~A}$ and $15 \mathrm{~V}$.

After conducting proper technical evaluation and user experiments on the thermal sweet taste device, we will be exploring proper stimulation parameters for the digital sweet taste reproduction. We hope in the near future, we will be able to develop a combined interface where we can effectively regenerate sour and sweet tastes digitally. In the medical industry this research will be able to provide mental pleasure for patients who suffer from clinical conditions such as diabetes.

\section{Magnetic interfaces}

\section{Magnetic table and magnetic foods}

In this research we are presenting a magnetic object levitation platform with a haptic display where users can interact with objects with changing weights and foods that are edible with magnetic properties.

The magnetic table interface will be developed with an array of electromagnets underneath of the table, where we can levitate / move the magnetic objects (utensils/foods) placed on top of its surface. The proposed platform is shown in Figure 11. This platform utilizes the fundamental physics of magnetic forces at a distance for object levitation. The force produced will repel, attract, vibrate or levitate the object. One of the main experiments we intend to do using this system is to study the effects of perceived smell and taste of food through weight of utensils. We will configure the proposed platform as a dining table and change the weight of utensils by using magnetic fields while eating.

Magnetic Table interface needs to generate very high magnetic field to move and levitate stuffs and therefore we will be using Bitter plate electromagnets [30]. Bitter electromagnets are known for generating extremely strong magnetic fields. In general, the field generated by Iron core electromagnets is often limited around 2 Teslas [31-33], while Bitter coils can produce very high magnetic field such as 45 Tesla. These electromagnets are made by attaching metal plates (Eg. Copper) and insulating plates on top of each other forming a helix type structure. This method enables the electromagnet to withstand the immense Lorentz forces created internally. Liquid coolant based cooling mechanism is needed for these electromagnets to absorb the heat generated and preventing them from melting down while operating.

Further, this interface would be able to create different types of haptic sensations using 'like polarity haptics'. Those haptic sensations can be felt like attraction, repulsion and various patterns of vibrations. Those sensations can be easily controlled by changing the polarity and frequency of the electromagnets. This technology will be able to provide interactions such as haptics in mid-air, guide the user to a particular location through a virtual path, provide surface features of $2 \mathrm{D}$ and $3 \mathrm{D}$ virtual objects, and restrict user to a certain virtual boundary. This magnetic table could also be used as new type of physical interaction display.

In future we are aiming to introduce specially created edible magnetic foods to use with this interface. The most iron enriched foods in the market can be movable using the electromagnets and we will be using iron and other type of metals that are reactive with external magnetic fields to make the foods. Also we will be testing the foods that have permanent magnet inside which will be something similar to a fruit or vegetable with a seed(s) inside. 


\section{Discussion}

We expect our digital smell and taste interfaces will open up a multitude of new horizons and opportunities for research in the future, including in areas of human computer interfaces, entertainment systems, medical and wellness. Digital controllability of the sensation of taste and smell provides a useful platform for engineers, food designers, and media artists towards developing multisensory interactions remotely, including the generation of new virtual tastes and smells for entertainment systems. For scholarly research, this would help bring about answers to exactly what is language of taste and smell.

The aim of our final prototypes is to develop them as portable and wearable units. Therefore, enabling users to wear those interfaces in daily lifestyle situations for augmentation. For example, Google Glasses promises a better experience of augmented reality unattainable with a smart phone. We can also realize accessories that would be attached to smart phones that could actuate a taste and smell by placing the end of the apparatus in one's mouth and/or nose. A friend could send you a taste or smell over the internet by means of a social network and you could taste or smell it electronically. They would simply input some sort of taste or smell into the phone by text means or by selecting presets related to their current experience.

Further these devices will be able to provide stimulation of a taste and smell from something that can't exist. This could also lead to a breakthrough in molecular gastronomy. Training molecular gastronomers is very difficult for most people, due to the requirement to be trained directly by experts at special facilities. Digital taste and smell systems could facilitate this training through a computer or over the internet. It could train one's sense of taste and smell and possibly lead to the creation of new kinds of consumer edible delights by quickly prototyping recipes.

We also believe patients who can't consume certain chemicals such as those with diabetes can stimulate the certain chemical in question (like sugar) taste in place of the actual chemicals. Our system could do so by augmenting pre-existing tastes like seasonings without the danger of the actual chemical being present in the food. It may also be possible to cancel out certain tastes that people wouldn't like, for instance bitterness, by adding more sugar to it, without worrying about consuming too much sugar. This might allow humans to even consume certain foods that were previously unappetizing to eat or to encourage children to eat unpopular foods to maintain a healthy lifestyle.

As an input idea, people could experience flavors and the computer could output musical beats for both amusement and recording the notes to store our recipes in an interesting way. In this scenario, taste can be composed like a song. They can be composed to vary over time, for example, a sudden change of taste from salty to sweet, which cannot be achieved using real foods. People can make new kinds of recipes using purely digital compositions and post them on a social media site.

Users can create new knowledge using a digital taste and smell machines and add pages embedded with the flavors similarly to adding pictures or sound to a webpage. People can rate the experiences by sharing them through another flavor. These experiences can gather to form more and more complex flavors and realize new potentials in gastronomy through our device. A chef or restaurant owner could introduce a new menu by sending alerts to fans of their work containing flavor information for new dishes. When the user opens the message, he or she can taste and smell the new menu from their accessory as well as the food image.

\section{Conclusion}

Audio, visual, and haptic fields are well researched. We believe the next challenge is virtual taste and smell using techniques that are currently unattainable using chemical based methods. Digital taste and smell technologies is one such attempt of actuating taste and smell with electric, thermal and magnetic stimulation noninvasively. This has advantages over chemical stimulation because it is more manageable and taste and smell can be transmitted directly over the internet. We believe through this research we can eventually digitally actuate all main tastes (bitterness, saltiness, sourness, sweetness, umami) and all main smells (putrid, vegetable, floral, woody, minty, fruity) using digital means to actuate and transmit the taste and smell information. It could have long term implications in the future of many disciplines. By using digital taste and smell, we will be able to create new ways to create content and new knowledge of food that can be shared, learned, and created in new interesting ways previously unobtainable with current methods.

\section{Acknowledgments}

We would like to thank our previous research contributors and all the members of the Imagineering Institute, Malaysia for the support they provided and all the previous researchers who worked with us for the earlier research works.

\section{References}

1. Volta A (1800) On the electricity excited by the mere contact of conducting substances of different kinds. In a letter from Mr. Alexander Volta, FRS Professor of Natural Philosophy in the University of Pavia, to the Rt. Hon. Sir Joseph Banks, Bart. KBPRS. Philosophical transactions of the Royal Society of London, pp.403-431.

2. Plattig KH, Innitzer J (1976) Taste qualities elicited by electric stimulation of single human tongue papillae. Pflugers Arch 361: 115-120. [Crossref]

3. Price S, Desimone JA (1977) Models of taste receptor cell stimulation. Chem Senses 2: 427-456.

4. Lawless HT, Stevens DA, Chapman KW, Kurtz A (2005) Metallic taste from electrical and chemical stimulation. Chem Senses 30: 185-194. [Crossref]

5. Lindemann B (1996) Taste reception. Physiol Rev 76: 718-766. [Crossref]

6. Ellegård EK, Goldsmith D, Hay KD, Stillman JA, Morton RP (2007) Studies on the relationship between electrogustometry and sour taste perception. Auris Nasus Larynx 34: 477-480. [Crossref]

7. Nakamura $\mathrm{H}$ and Miyashita $\mathrm{H}$ (2011) Augmented gustation using electricity. In Proceedings of the 2nd Augmented Human International.ACM34.

8. Kumar G, Juhasz C, Sood S, Asano E (2012) Olfactory hallucinations elicited by electrical stimulation via subdural electrodes: effects of direct stimulation of olfactory bulb and tract. Epilepsy \& Behavior 24: 264-268.

9. Yamamoto C (1961) Olfactory bulb potentials to electrical stimulation of the olfactory mucosa. Jpn J Physiol 11: 545-554. [Crossref]

10. Ishimaru T, Shimada T, Sakumoto M, Miwa T, Kimura Y, et al. (1997) Olfactory evoked potential produced by electrical stimulation of the human olfactory mucosa. Chemical senses 22: 77-81.

11. Ishimaru T, Miwa T, Shimada T, Furukawa M (2002) Electrically stimulated olfactory evoked potential in olfactory disturbance. Annals of Otology, Rhinology \& Laryngology 111: 518-522.

12. Doty RL (1997)Practical approaches to clinical olfactory testing. Taste and Smell Disorders. Thieme : New York, 38-51.

13. Kortum P (2008) HCI beyond the GUI: Design for haptic, speech, olfactory, and other nontraditional interfaces. Morgan Kaufmann.

14. Maynes-Aminzade D (2005) Edible bits: Seamless interfaces between people, data and food. In Conference on Human Factors in Computing Systems (CHI'05)-extended abstracts 2207-2210.

15. Edition.cnn.com (2015) Designers developing virtual-reality 'Cocoon' - CNN.com. 
16. Renaud B, Buda M, Lewis BD, Pujol JF (1975) Effects of 5,6-dihydroxytryptamine on tyrosine-hydroxylase activity in central catecholaminergic neurons of the rat. Biochem Pharmacol24: 1739-1742. [Crossref]

17. Talavera K, Yasumatsu K, Voets T, Droogmans G, Shigemura N, et al. (2005) Heat activation of TRPM5 underlies thermal sensitivity of sweet taste. Nature 438: 10221025. [Crossref]

18. Cruz A, Green BG (2000) Thermal stimulation of taste. Nature 403: 889-892. [Crossref]

19. Suzuki C, Narumi T, Tanikawa T, HiroseM (2014) Affecting tumbler: affecting our flavor perception with thermal feedback. InProceedings of the 11th Conference on Advances in Computer Entertainment Technology.ACM19.

20. Grinder J,Bandler R (1993) Tranceformations. Real People Press 5.

21. Jansen Y, Karrer T, Borchers J (2010) Mudpad: tactile feedback and haptic texture overlay for touch surfaces. In ACM International Conference on Interactive Tabletops and Surfaces. $A C M 11: 23$

22. Weiss M, Wacharamanotham C, Voelker S, Borchers J (2011) Fingerflux: near-surface haptic feedback on tabletops. In Proceedings of the 24th annual ACM symposium on User interface software and technology (New York, NY, USA,), UIST '11.ACM5: 615620

23. Wakita A, Nakano A, Kobayashi N (2011) Programmable blobs: a rheologic interface for organic shape design. In Proceedings of the fifth international conference on Tangible, embedded, and embod-ied interaction. ACM 5: 273-276.

24. Frey M (2006) Snoil. a physical display based on ferrofluid. 5: 22.
25. Hook J, Taylor S, Butler A, Villar N, Izadi S (2009) A reconfigurable ferromagnetic input device. In Proceedings of the 22nd annual ACM symposium on User interface software and technology.

26. Cameron C, Zhao H, McHugh MK (2012) Perspective: publication ethics and the emerging scientific workforce: understanding "plagiarism" in a global context. Acad Med 87: 51-54. [Crossref]

27. Karunanayaka K, Koh JTKV, Naik EB, Cheok AD (2011) Hall Effect sensing input and like polarity haptic feedback in the liquid interface system. In Ambient Intelligence. Springer Berlin Heidelberg pp, 141-145.

28. Holman D, Vertegaal R (2008) Organic user interfaces: designing computers in any way, shape, or form. Communications of the ACM 51: 4855

29. Karunanayaka K, Siriwardana S, Edirisinghe C, Nakatsu R, Gopalakrishnakone P (2013) Haptic Mouse Magnetic Field Based Near Surface Haptic and Pointing Interface. Int.Journal of In-teractive Digital Media 1: 8-15.

30. Jones J (2008) Nose and Nasal cavities.

31. Beauchamp GK, Cowart BJ (1987) Development of sweet taste. In Sweetness. Springer London.pp. 127-140.

32. Geim A (1998) Everyone's magnetism. Physics Today51: 36-39.

33. High Temprature Superconductivity in Perspective (1990) US Government Printing Office:Washington DCp. 42.

Copyright: (C2016 Karunanayaka KT. This is an open-access article distributed under the terms of the Creative Commons Attribution License, which permits unrestricted use, distribution, and reproduction in any medium, provided the original author and source are credited. 